\title{
Coronary Heart Disease: Risk Factors as Guides to Preventive Action
}

\author{
JEREMIAH STAMLER \\ Department of Community Health and Preventive Medicine, \\ Northwestern University Medical School, Chicago, Illinois, 60611 \\ AND \\ Frederick H. EPSTEIN ${ }^{1}$ \\ Department of Epidemiology, School of Public Health, \\ and the Center for Research in Diseases of the Heart, \\ University of Michigan, Ann Arbor, Michigan 48104
}

Candidates for future development of premature coronary heart disease (CHD) can now be identified with a degree of probability unsurpassed for most other diseases, and equaled for few. This remarkable achievement has not come about fortuitously, but through the deliberate planning of long-term epidemiological studies.

The ultimate purpose of defining risk in quantitative terms is the identification of susceptibles in the community, in order to institute preventive measures. The predictability of coronary disease in middle age from simple risk factor measurements provides an invaluable scientific base for curbing the contemporary epidemic of this preeminent chronic disease. The eventual elimination of this epidemic demands, in addition to identification and care of susceptibles, changes in the mode of life in the national community at large $(1,2)$.

\section{HYPERCHOLESTEROLEMIA, HYPE'TE'ESION, AND CIGARE'I"IE SMOKING}

The three risk factors - hypercholesterolemia, hypertension and cigarette smoking-have not been "discovered" through epidemiological studies. Rather, clinicians and researchers have known or suspected for a long time that they play an etiological role in coronary heart disease. Epidemiological investigations have proven the associations and put them into quantitative terms. These three predictors . . are properly designated major risk factors for premature atherosclerotic dis-
ease, especially coronary disease. This designation is appropriate, first because of
the impact of these factors on risk, particularly when present in combination, second
because of the consistency of the findings from multiple studies, and third because
of the frequency of occurrence of these factors singly and in combination in
the American population. All three are potentially amenable to prevention and
control (1).

\footnotetext{
${ }^{1}$ Recipient of Career Research Award HE-K6-6748 from the National Heart and Lung Institute, National Institutes of Health, Bethesda, MD.
}

(C) 1972 by Academic Press, Inc. 

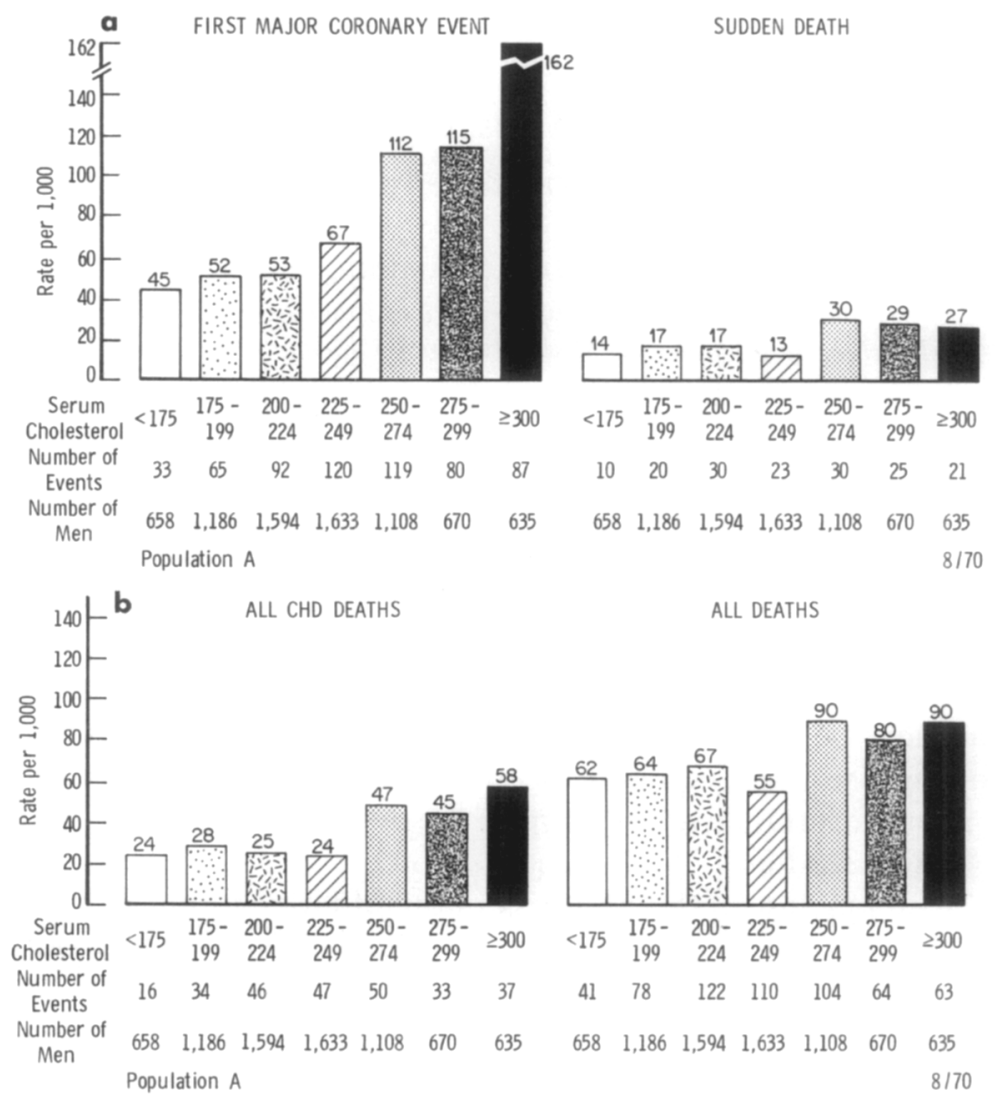

Fig. 1. National cooperative Pooling Project; serum cholesterol level at entry and 10-year ageadjusted rated per 1,000 men for: any major coronary event, sudden death $(1 a)$; any coronary death, death from all causes ( $1 b$ ). Any major coronary event includes nonfatal MI, fatal MI, sudden death due to $\mathrm{CHD}$; U. S. white males age 30-59 at entry; all rates age-adjusted by 10-year age groups to the U.S. white male population, $1960(6,6 a-1)$.

Of course, these three are not the only coronary risk factors, and in a given individual others may assume greater importance. However, no other variables presently known are equally effective in population groups for identifying persons prone to develop manifest disease over the next 5 or 10 years. That is, these three factors, in combination, concentrate the majority of subsequent new events of coronary disease among a minority of the population at risk. Their preeminence as predictors is confirmed by multivariate analysis (see below), using a series of additional factors and testing their relative importance by a modified form of discriminant analysis (1-7).

In Figs. 1-3, the three variables have first been treated singly. ${ }^{2}$ In agree-

${ }^{2}$ These are preliminary data from the national cooperative Pooling Project $(6,6 \mathrm{a}-1)$. In this Project, data on risk factors collected at initial examination, and 10-year follow-up data on disease incidence and mortality, have been pooled from several major U.S. prospective studies. The base population for the figures presented here is derived from five studies, and numbers 7,594 white males age 30-59 and free of signs of definite CHD at entry. 
ment with the findings from most U. S. prospective studies, risk of developing premature CHD -including sudden death and all fatal CHD-increases as the serum cholesterol rises (Fig. $1 a, b)(1)$. Since CHD is the single most important cause of death, mortality from all causes is also related to cholesterolemia. For first major coronary events (nonfatal and fatal myocardial infarction plus sudden deaths attributed to CHD), there is an almost fourfold gradient in risk between those at opposite ends of the range, below 175 and above $299 \mathrm{mg} / \mathrm{dl}$. There are fewer than $10 \%$ of the men in these two categories. Note that almost half of the new events (286 out of 596) occur among the third of the men with levels of $250 \mathrm{mg} / \mathrm{dl}$ or above. Note also that this upper third had a level of serum cholesterol carrying a 10-year CHD risk about twice as high as that seen among the remainder of the population (119/1000 as opposed to 61$)$.

The findings for blood pressure and these same end points are similar (Fig.
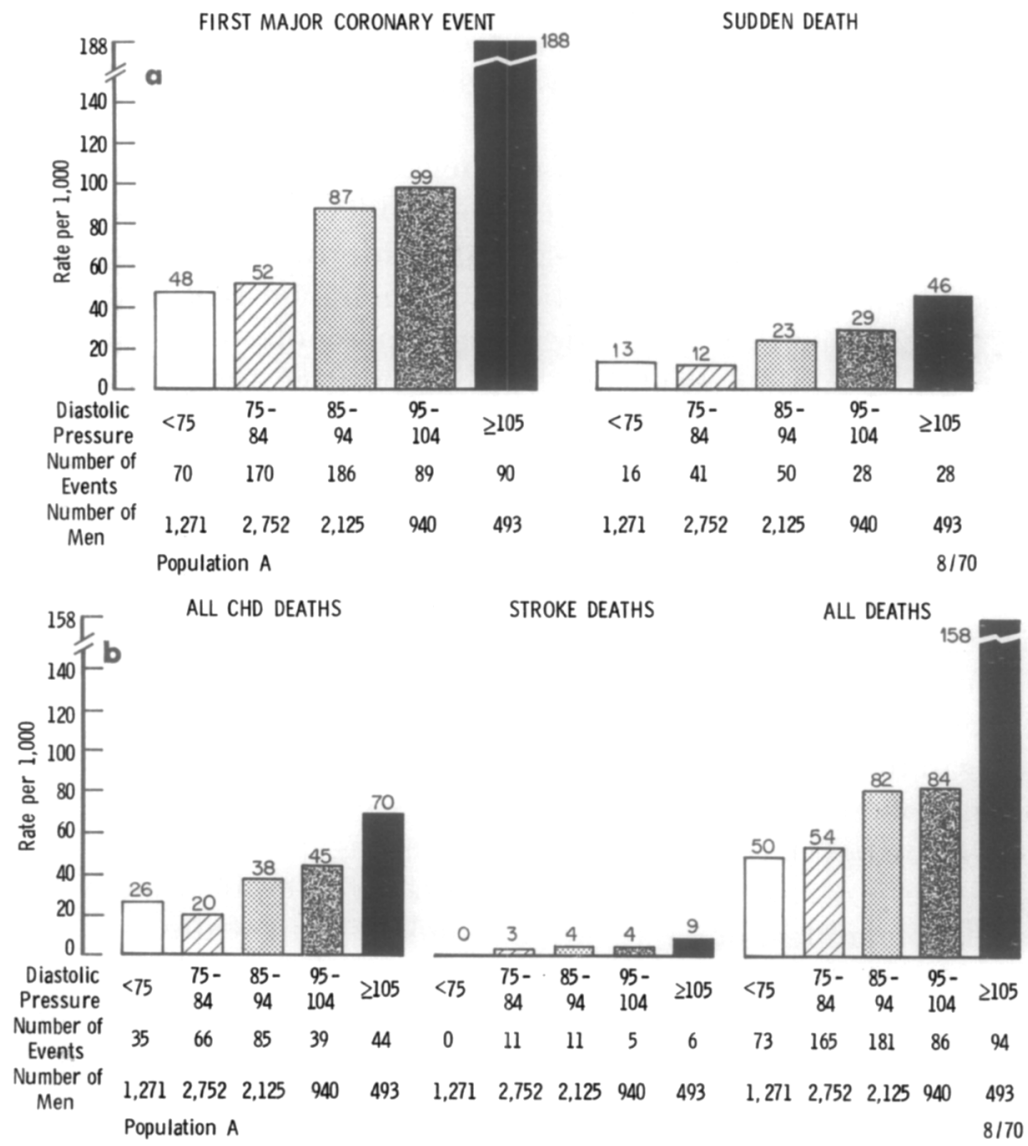

FIG. 2. National cooperative Pooling Project; diastolic blood pressure level at entry and 10-year age-adjusted rates per 1,000 men for: any major coronary event and sudden death $(2 a)$; any coronary death, stroke death, death from all causes $(2 b)$. Any major coronary event includes nonfatal MI, fatal MI, sudden death due to CHD; U. S. white males age 30-59 at entry; all rates ageadjusted by 10 -year age groups to the $U$. $S$. white male population, $1960(6,6 \mathrm{a}-1)$. 
$2 a, b)(1)$. While Fig. 2 deals only with diastolic pressure, essentially the same results are available for systolic pressure. Compared to optimal levels of blood pressure, even "modest" elevations (i.e., diastolics of 85-94 or 95-104 mm $\mathrm{Hg}$ ) are associated with gross increases in risk - a point of great practical significance for clinicians.

To put this important observation in a more general way, for each of these quantitative variables, there is a steady increment in risk as level of the variable rises. As cholesterol concentration-or blood pressure-increases, risk increases. The relationships are continuous. There is no evidence of a critical level which divides "normal" subgroups (i.e., subgroups "immune" to premature coronary heart disease) from CHD-prone "abnormal" subgroups. For individuals, this conclusion from the massive epidemiologic data can be reformulated as follows: The higher the risk factor level, the greater the probability for an individual of developing coronary disease. This is important from the point of view of instituting preventive measures. The greater the probability, the greater the need for prophylaxis-but there is no single "screening level" separating those in need of prophylaxis from those who are not.

This basic set of conclusions does not negate-but rather places in proper context - the clinical use of practical cutting points, e.g., serum cholesterol of less than $200 \mathrm{mg} / \mathrm{dl}$ as normal; 200-249 as borderline, 250 or greater as abnormal. As American Heart Association statements on risk factors have emphasized, this $250 \mathrm{mg} / \mathrm{dl}$ level for defining hypercholesterolemia is approximately the 2 to 1 cutting point, i.e., persons positive for this risk factor are approximately twice as susceptible to premature CHD as those with lower levels (everything else being equal). The impact of these factors is no small $10 \%$, but rather $100 \%-$ a doubling of risk. But as useful as this practical approach of cutting points is, it remains a distortion of rcality. After all, a person with a serum cholesterol of 240 is at greater risk than one at 210 and he in turn is at greater risk than one at 160 . How then to make use of all the quantitative information in clinical and public health practice? New methods of statistical evaluation do in fact make this possible (see below).

As for cigarette smoking, massive studies in the United States have repeatedly shown that any regular use of cigarettes is associated with increased risk of CHD (Fig. 3a,b) (1). However, the risk for ex-cigarette smokers is about the same as for those who never smoked. The practical implications are obvious: Don't smoke cigarettes, or-if a current smoker-quit while still ahead, i.e., still free of CHD. It pays!

For those regularly smoking cigarettes when first examined, risk rose steadily with number of cigarettes smoked per day (Fig. 3). Once again, the relationship is quantitative and continuous. The gradient is twofold between those who smoke a pack or less and those smoking more than a pack. A "doseresponse" relationship of this kind is one piece of evidence in favor of a causative relationship. (Another is the consistent finding in several postmortem studies that coronary atherosclerosis was more severe in persons who had smoked cigarettes than in non-cigarette users.) Note in Fig. 3 that $60 \%$ of the 


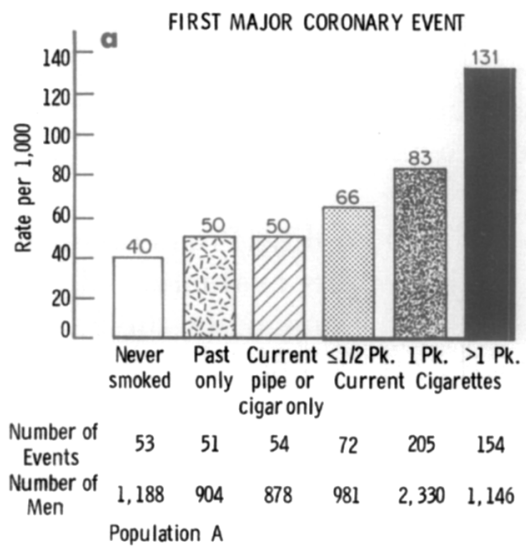

SUDDEN DEATH
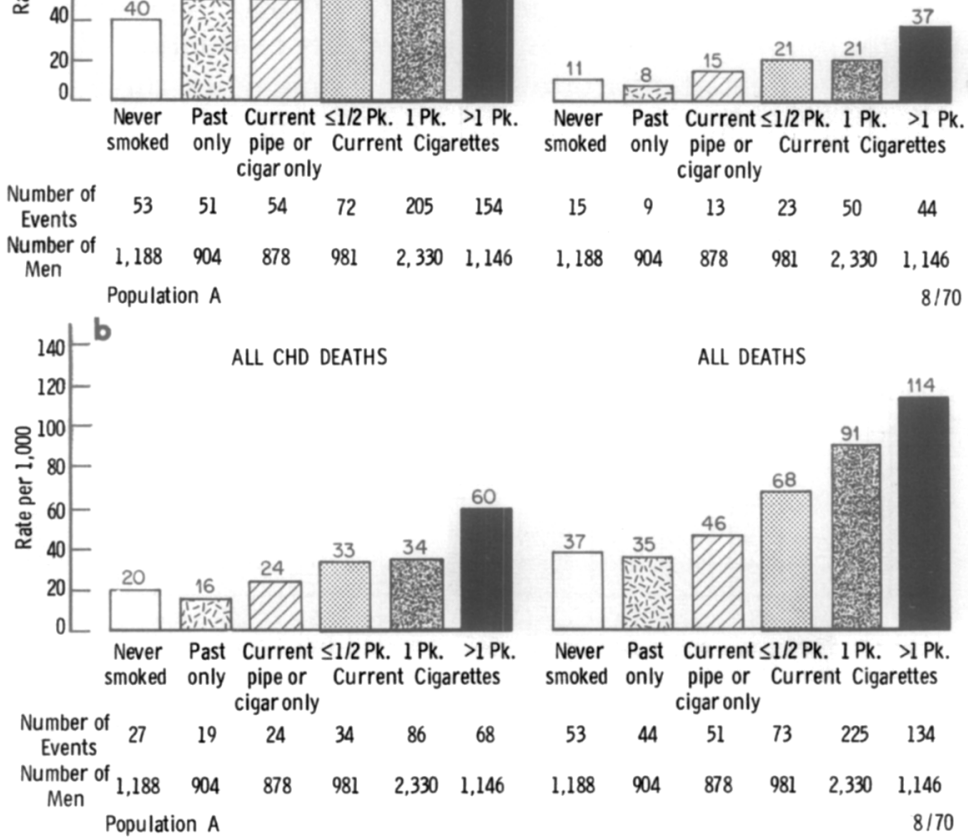

ALL DEATHS

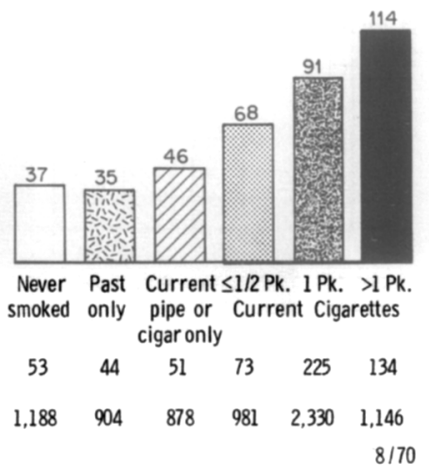

FIG. 3. National cooperative Pooling Project; smoking status at entry and 10-year age-adjusted rates per 1,000 men for: any major coronary event, sudden death, any coronary death, death from all causes: any major coronary event includes nonfatal MI, fatal MI, sudden death due to CHD; U.S. white males age 30-59 at entry; all rates age-adjusted by 10 -year age groups to the U. S. white male population, 1960 . Graphs present smoking status at entry and the 10-year age-adjusted rates, irrespective of other risk factors $(6,6 \mathrm{a}-\mathrm{l})$.

men are cigarette smokers. They account for no less than 431 out of the 589 new events (73.2\%)! It will be most interesting to have a clinical look at the 53 non-smokers (never smoked) with new events. How many of them had high serum cholesterol and/or blood pressure? This last question highlights the need to look at combinations of risk factors simultaneously, as shown next.

In Fig. $4 a-d$, the three risk factors are arranged in various combinations, in order to assess their additive or synergistic predictive power (1). In this display, a single cutting point has reluctantly been used to separate "high" from "not high" levels. (N. B.: "Not high" is not equal to low or optimal-at least not for serum cholesterol and diastolic pressure, as is evident from the cutting points of $250 \mathrm{mg} / \mathrm{dl}$ and $90 \mathrm{~mm} \mathrm{Hg}$, respectively.) In view of what has just been said, this approach is arbitrary and undesirable. However, such analyses would become impossibly complex if the variables were quantitated at several levels. As long as this limitation is kept in mind, looking at combinations of risk factors in this way is useful and informative. As already noted, 
newer biomathematical techniques, specifically multivariate risk functions (see below), obviate these problems by treating risk factors as continuous, strictly quantitative variables. However, this approach has not yet been translated into clinical terms. There is a place, therefore, for both kinds of display.

In Figs. $4 a$ and $b$, the entire group of 7,342 Pooling Project men are divided into six of eight possible subgroups. This permits evaluation of the independent and additive effect of cigarette smoking. Thus, when the 2,018 men with cigarette smoking as the only risk factor are compared with the 1,249 men

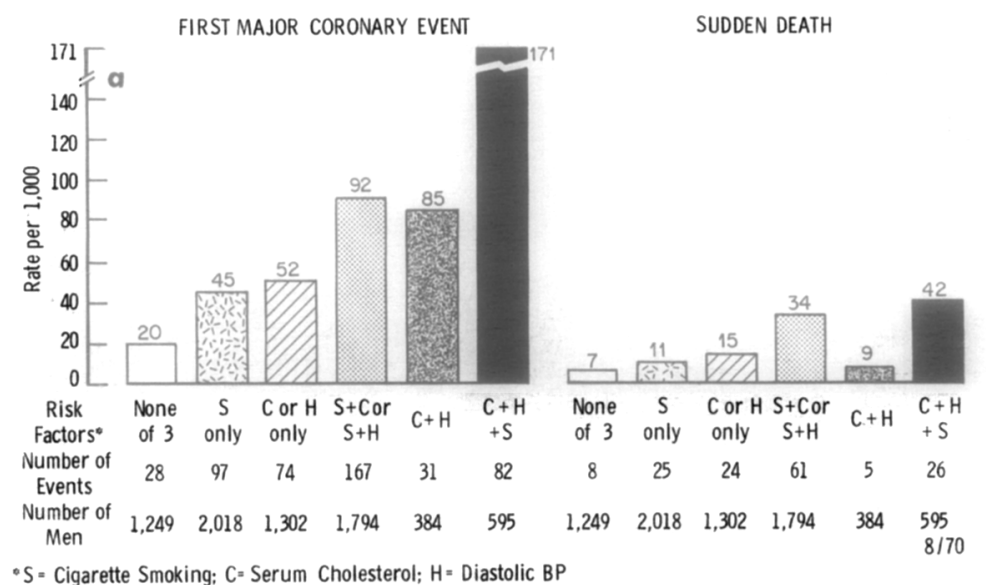

- $\mathrm{S}=$ Cigarette Smoking; $\mathrm{C}=$ Serum Cholesterol; $\mathrm{H}=$ Diastolic BP

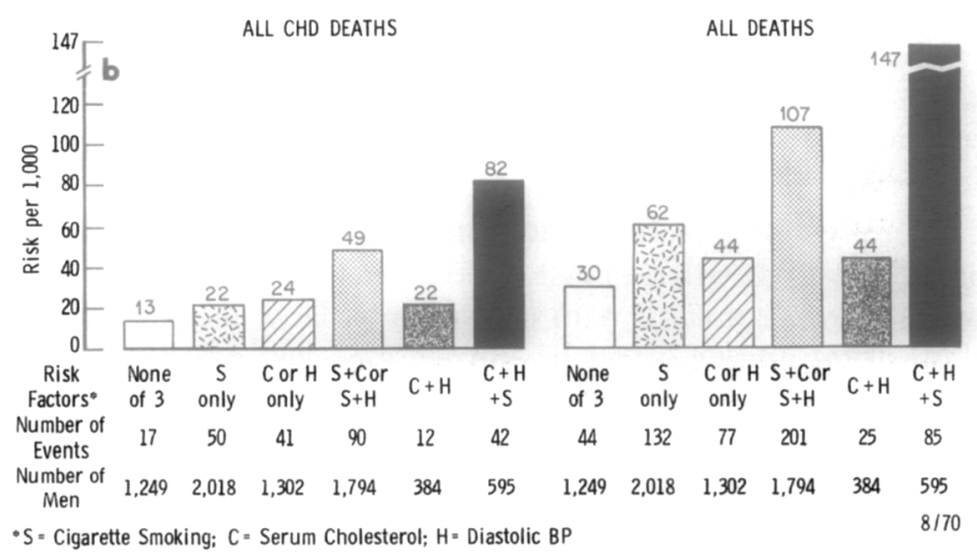

FIG. 4a,b. National cooperative Pooling Project; smoking status at entry and 10-year age-adjusted rates per 1,000 men for: any major coronary event, sudden death, any coronary death, death from all causes; any major coronary event includes non-fatal MI, fatal MI, sudden death due to CHD; U. S. white males age 30-59 at entry; all rates age-adjusted by 10-year age groups to the U. S. white male population, 1960. Graphs present rates for non-cigarette smokers vs. cigarette smokers at entry, with simultaneous control of blood pressure and serum cholesterol level; for this analysis, the following cutting points were used: cigarette smoking (S) any use at entry, serum cholesterol $(C) \geqslant 250 \mathrm{mg} / \mathrm{dl}$, diastolic blood pressure $(\mathbf{H}) \geqslant 90 \mathrm{~mm} \mathrm{Hg}(6,6 \mathrm{a}-\mathrm{l})$. 

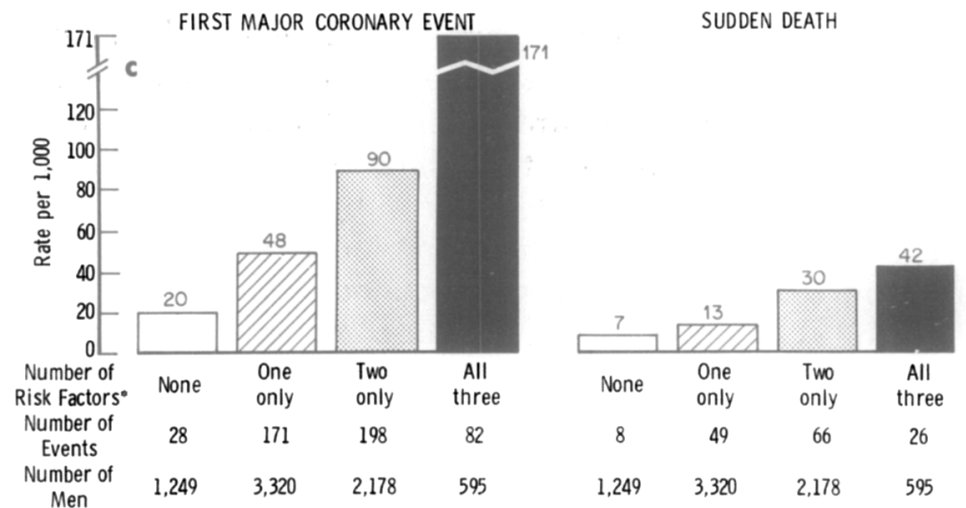

-Cutting Points: Serum Cholesterol $\geq 250 \mathrm{mg}$./ dl.; Diastolic BP $\geq 90 \mathrm{~mm}$. $\mathrm{Hg}$; Any use of Cigarettes at entry; Population A

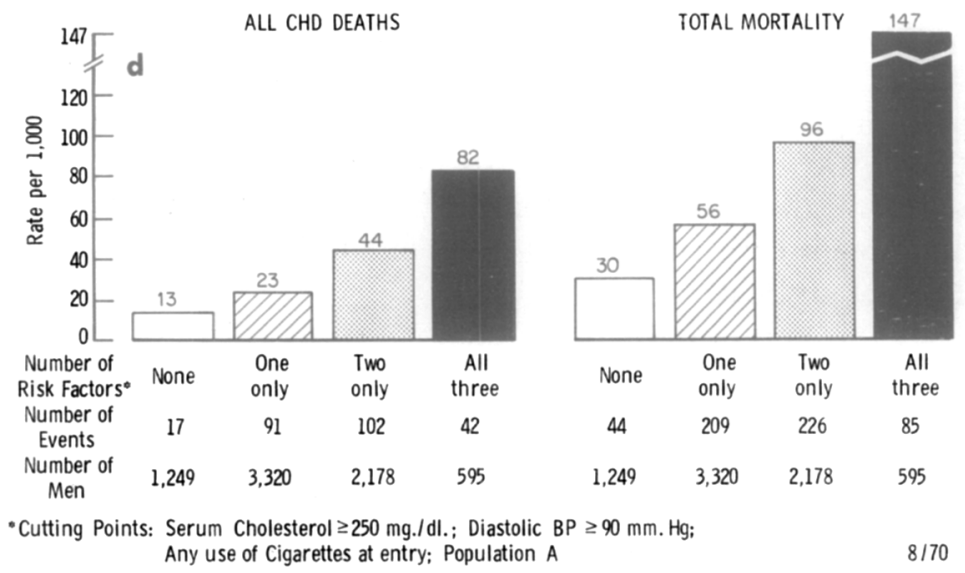

FIG. 4c,d. National cooperative Pooling Project; hypercholesterolemia, hypertension, cigarette smoking and 10-year age-adjusted rates per 1,000 men for: any major coronary event, sudden death (4c), any coronary death, death from all causes (4d); any major coronary event includes nonfatal MI, fatal MI, sudden death due to CHD; U. S. white males, age 30-59 at entry; all rates ageadjusted by 10 -year age groups to the U. S. white male population, $1960(6,6 \mathrm{a}-\mathrm{l})$.

with none of the three risk factors, the 10-year rates of first major coronary events are 45 and 20 per 1,000, respectively. Cigarette smoking is associated with a more than twofold increase in risk. The situation is similar for the other two paired comparisons - cigarette smokers vs. non-cigarette smokers, with one other risk factor (1,794 and 1,302 men, respectively); cigarette smokers vs. non-cigarette smokers, with two other risk factors (595 and 384 men, respectively). Moreover, cigarette smoking consistently makes an independent and additive contribution to risk of sudden death, coronary death, and death from all causes.

Similar analyses also demonstrate that serum cholesterol and blood pressure-like cigarette smoking - each make an independent and additive contribution to risk.

In Fig. $4 c$ and $d$, the same data are again displayed, in simpler form, so that 
the three risk factors are not distinguished one from the other. Once again it is worth noting: the analysis is a crude one, in that each man is characterized only on the basis of a single measurement of each risk factor at the entry examination, and his status with respect to each factor is arrived at by dichotomization (high or not high). Nevertheless the subgroups were very different in their 10-year morbidity and mortality experience. Presence of only one risk factor-as compared to none-was associated with a substantial increase (2.4-fold, or $140 \%$ ) in probability of a major coronary event over the next decade. Increase in risk of a fatal event, including total mortality, was almost double. For the men with any two of the risk factors, risk of a major event was increased more than fourfold; risk of death, more than threefold. For the men with all three factors, risk of a major event was increased more than eightfold; risk of death, fivefold. This is indeed powerful prediction from three simple variables, measured once, and simply dichotomized-far more powerful prediction than is available for most other diseases, acute or chronic!

Note further the typical American situation, product of our way of life: only 1,249 of the 7,342 white males age 30-59 at entry-i.e., only $17 \%$ - were classified not high for all three factors. All the rest had one or more risk factors: $45 \%$ with one, $30 \%$ with two, $8 \%$ with all three. These latter two subgroups, with any two or all three risk factors (38\% of the total group), accounted for $58 \%$ of first major coronary events, $62 \%$ of sudden deaths, $57 \%$ of coronary deaths, $55 \%$ of all deaths.

Obviously it is appropriate to designate persons with such combinations of these traits as very high risk individuals, very prone to premature atherosclerotic disease. Obviously too, especially since a first event is so often a catastrophic one ( $25 \%$ are sudden deaths), it is very sound strategy to detect such very high risk men as early in young adulthood and middle age as possible, and to asșist them preventively by bringing about sustained correction of their risk factors, by safe well-tested means - nutritional for hypercholesterolemia, hygienic for cigarette smoking, nutritional (weight control and moderate salt restriction) plus pharmacologic (when necessary) for hypertension.

A halfing of the CHD mortality rate by such means in the subgroup of Pooling Project men with any two or all three of these risk factors - a reasonable goal, from a rate 4 times that of the subgroup with no risk factors to a rate still twice as high - would have saved 72 lives. For the entire Pooling Project group of 7,342 men, this would have meant a reduction in CHD mortality rate of $29 \%$ - and a reduction in mortality rate from all causes of $13 \%$. Project this to the U. S. population of young and middle-aged male adults as a whole-with almost 150,000 coronary deaths per year prior to age 65 . The saving would be 40,000 lives per year! The reduction in total mortality rate would be about equal to that achicved for adult malcs by all other means since 1900 ! This is the meaning - this is the stake - in the "risk factor story"!

PREDICTING RISK FROM MULTIVARIATE FUNCTION

As already noted, serious limitations exist in the cross-classification method for evaluating impact of combinations of risk factors. When only three factors 
are considered simultaneously and each is crudely evaluated by simple dichotomization, as above, the population at risk is divided into cight groups. When four variables are simultaneously considered in a similar fashion, the number of cells increases to 16 ; with five variables, to 32 . Under these analytical circumstances, several cells become too small in size, even with quite a large population available for study, as in the Pooling Project. Moreover, dichotomization - by treating each and every level of a risk factor as either high or not high-utilizes quantitative data in the most crude and meager fashion, rather than taking full advantage thereof.

In recent ycars, scvcral statistical methods for multivariate analysis - some of them newly developed-have been applied to this problem. In 1967, data utilizing one such method-the multiple logistic model-were published from the Framingham study, based on the risk factor findings at first examination and 12-year CHD incidence (3). This method made it possible to compute a risk probability for each individual based on simultaneous evaluation of his several findings, e.g., serum cholesterol, blood pressure, smoking habit, etc.

For this purpose, use was madc of the statistical method of linear discriminant function to estimate coefficients for several risk factors considered simultaneously. The coefficients were obtained using the observed data from the Framingham population on risk factor levels and 12-year CHD incidence.

TABLE I

Linear Discriminant Function Coefficients $(\beta)$ and Constant $(\alpha)$, Men And WOMEN, AGe 30-62 AND FREE OF CHD AT ENTRY, BASED ON 12-Year Follow-up Data ON CHD INCIDENCE,

The FRamingham STUdy (3)

\begin{tabular}{|c|c|c|c|c|}
\hline \multirow{2}{*}{$\begin{array}{l}\text { Risk factors (x's) } \\
\text { - findings on } \\
\text { initial } \\
\text { examination }\end{array}$} & \multicolumn{2}{|c|}{ 2,187 Men } & \multicolumn{2}{|c|}{ 2,669 Women } \\
\hline & Coefficient & $\begin{array}{l}\text { Standard error } \\
\text { of estimated } \\
\text { coefficient }\end{array}$ & Coefficient & $\begin{array}{l}\text { Standard error } \\
\text { of estimated } \\
\text { coefficient }\end{array}$ \\
\hline Age (Years) & 0.0708 & 0.0083 & 0.0765 & 0.0133 \\
\hline $\begin{array}{l}\text { Serum Cholesterol } \\
(\mathrm{mg} / \mathrm{dl})\end{array}$ & 0.0105 & 0.0016 & 0.0061 & 0.0021 \\
\hline $\begin{array}{l}\text { Systolic BP } \\
\text { (mm Hg) } \\
\text { Framingham }\end{array}$ & 0.0166 & 0.0036 & 0.0221 & 0.0043 \\
\hline $\begin{array}{l}\text { Relative Weight } \\
\text { Hemoglobin }\end{array}$ & 0.0138 & 0.0051 & 0.0053 & 0.0054 \\
\hline$(\mathrm{gm} / \mathrm{dl})$ & -0.0837 & 0.0542 & 0.0355 & 0.0844 \\
\hline $\begin{array}{l}\text { Cigarettes/day }{ }^{a} \\
\text { ECG }\end{array}$ & 0.3610 & 0.0587 & 0.0766 & 0.1158 \\
\hline Abnormality ${ }^{b}$ & 1.0459 & 0.2706 & 1.4338 & 0.4342 \\
\hline Constant $(\alpha)$ & -10.8986 & - & -12.5933 & - \\
\hline
\end{tabular}

${ }^{a}$ Coded as: $0=$ never smoked; $1=<$ pack $/$ day; $2=1$ pack $/$ day; $3=>$ pack $/$ day.

${ }^{\circ}$ Coded as: 0 for normal; 1 for definite or possible LVH, definite nonspecific abnormality and intraventricular block. 
TABLE II

EXPECTED AND OBSERVED NUMBER OF CASES OF CHD AND

OBSERVED INCIDENCE IN 12 YeARS OF FOLLOW-UP,

MEN AND WoMen AGE 30-62 and Free of CHD at ENTRY,

The Framingham Study (3)

\begin{tabular}{ccccccc}
\hline & \multicolumn{3}{c}{2,187 Men } & \multicolumn{3}{c}{2,669 Women } \\
\cline { 2 - 7 } $\begin{array}{c}\text { Decile of Risk } \\
\text { calculated from } \\
\text { multiple logistic }\end{array}$ & $\begin{array}{c}\text { No. of } \\
\text { cases } \\
\text { expected }\end{array}$ & $\begin{array}{c}\text { No. of } \\
\text { cases } \\
\text { observed }\end{array}$ & $\begin{array}{c}\text { Observed } \\
\text { 12-year } \\
\text { incidence } \\
\text { (per 1,000) }\end{array}$ & $\begin{array}{c}\text { No. of } \\
\text { cases } \\
\text { expected }\end{array}$ & $\begin{array}{c}\text { No. of } \\
\text { cases } \\
\text { observed }\end{array}$ & $\begin{array}{c}\text { Observed } \\
\text { 12-year } \\
\text { incidence } \\
\text { (per 1,000) }\end{array}$ \\
\hline 1 & 3.4 & 0 & 0 & 1.1 & 1 & 4 \\
2 & 6.0 & 3 & 14 & 1.7 & 3 & 11 \\
3 & 8.6 & 10 & 46 & 2.3 & 0 & 0 \\
4 & 11.5 & 13 & 59 & 3.2 & 2 & 7 \\
5 & 15.0 & 20 & 91 & 4.4 & 6 & 22 \\
6 & 19.7 & 22 & 101 & 6.5 & 5 & 19 \\
7 & 25.0 & 33 & 151 & 9.8 & 14 & 52 \\
8 & 32.6 & 31 & 142 & 15.0 & 21 & 79 \\
9 & 47.1 & 44 & 201 & 24.7 & 23 & 86 \\
10 & 90.5 & 82 & 375 & 70.4 & 54 & 202 \\
\hline All & 259.4 & 258 & 118 & 139.1 & 129 & 48 \\
\hline
\end{tabular}

The coefficients for males and females age 30-62 at entry are presented in Table I. The value for CHD risk $y$ for each individual was then computed according to the formula:

$$
y=1 /\left[1+e-\left(\alpha+\beta_{1} x_{1}+\beta_{2} x_{2} \ldots \beta_{7} x_{7}\right)\right]
$$

where $e$ is the base for natural or Naperian logarithms, i.e., equal to $2.71828 \ldots, \alpha$ is an estimated constant, each of the betas is an estimated coefficient, and each $x$ is a risk factor value (as measured at initial examination) (3). In the analysis of the Framingham data, the seven risk factors entered into the equation were: age, serum cholesterol, systolic blood pressure, cigarettes smoked per day, relative weight, hemoglobin, and ECG findings. For a given age-sex group, the calculated risk values $y$ of all the individuals were ranked in order from low to high, and deciles were then identified. For each decile, the number of predicted cases of CHD was then determined by summing the risks $y$ calculated from the equation for all the persons in the decile. For each decile, the actual number of observed CHD cases was also tallied, and the observed 12-year incidence rate calculated. These data for the 2,176 men and the 2,669 women age 30-62 and free of CHD at entry are presented in Table II (3). Obviously, the method yielded close correspondence in number of observed and expected cases. Obviously also, the method successfully graded both men and women in regard to risk. Based on the observed 12-year incidence rates, the ratios of risk for the upper two deciles compared with the lower are more than 40:1 for men and 19:1 for women. 
Of course, this result was to be expected, since the coefficients generated from the Framingham experience itself were then utilized to estimate risk of the individuals in this study. The key question really is the generalizability of these data. That is, how correctly do these coefficients-derived from the Framingham experience-predict risk of other individuals and other populations?

At least two reports are available on applying the risk function developed from the Framingham data to the experience of other prospective studies. The first was published as part of the recent monograph on 5-year incidence findings from the International Cooperative Study on the Epidemiology of Cardiovascular Disease (4). In this instance, the method was successfully applied for prediction of an expected rate for each of the several populations overall, and then for a comparison among them, i.e., for an interpopulation analysis. The correspondence between observed and expected rates for the several populations was indeed high, with a correlation coefficient of 0.83 .

Recently, this multiple logistic method and the coefficients from the Framingham study were applied to the data from the Peoples Gas Company study (7). For this purpose, use was made of the cohort of 1,211 white males age 40-59 and free of overt CHD at first examination in 1958. This age-race cohort is the group from the Peoples Gas Company study entered in the national cooperative Pooling Project. For this cohort, data are available concerning incidence of CHD over the first ten years of follow-up, from 1958-1968. Since the coefficients for the Framingham study had been generated based on the 12-year CHD incidence data, it was deemed most appropriate to apply them to the same end point for the Peoples Gas Company men.

With use of the Framingham coefficients, 12-year risk was computed for each Peoples Gas Company man, and the entire cohort was ordered by quintiles based on predicted risk. Since the Framingham data are based on 12 years of follow-up, rather than ten, the number of CHD events expected in each Peoples Gas Company quintile of risk over 12 years (as generated by the equation) was corrected by multiplying by $10 / 12$ or 0.8333 , for comparison with the actual number of events observed (Table III). As is evident, these calculations resulted in reasonably good correspondence between the numbers of expected and observed events for the quintiles. Fifty-nine of the 151 observed events $-39.1 \%$-occurred in the men classified predictively in the upper quintile of risk. Ninety-four of the 151 observed events $-62.3 \%$-occurred in the men classified in the upper two quintiles of risk. The ratio of risk for the highest quintile compared with the lowest was $5: 1$, i.e., predictive power was reasonably good.

These two initial experiences, international and national, on prediction in other populations with use of this method and the Framingham coefficients are encouraging, in regard to the key question of generalizability. As these methods are more extensively applied, using larger sets of risk factors and additional populations (e.g., those cooperating in the Pooling Project), ability to predict risk-i.e., the broad generalizability of these methods-should be enhanced. Practitioners of preventive medicine and public health can antici- 
TABLE III

APPLICATION OF COEFFICIENTS FROM FRAMINGHAM STUDY to Predict Ten-Year Risk of First CHD Events in

Peoples Gas Co. Cohort of White Males Age 40-59 and Free of OvERT CHD AT First EXAMINATION IN 1958 Multiple Logistic Method (5)

\begin{tabular}{ccccc}
\hline Quintile of risk & $\begin{array}{c}\text { No. of men } \\
\text { at risk }\end{array}$ & $\begin{array}{c}\text { No. of expected } \\
\text { events }\end{array}$ & $\begin{array}{c}\text { No. of observed } \\
\text { events }\end{array}$ & $\begin{array}{c}\text { Observed incidence } \\
\text { rate per 1,000 }\end{array}$ \\
\hline 1 & 241 & 10.9 & 12 & 50 \\
2 & 244 & 20.3 & 18 & 74 \\
3 & 242 & 31.9 & 27 & 111 \\
4 & 242 & 47.7 & 35 & 145 \\
5 & 241 & 88.1 & 59 & 245 \\
\hline All & 1,211 & 198.9 & 151 & 125 \\
\hline
\end{tabular}

pate that before long these new practical tools will be made generally available, for precise quantitative assessment of risk based on multiple risk factors. This should mean a further improvement over present approaches-already very useful-for identifying individuals highly susceptible to premature atheroselerotic disease.

HYPERTRIGLYCERIDEMIA-

HYPERLIPOPROTEINEMIA, HYPERGLYCEMIA, HYPERURICEMIA, OBESITY

Among the several serum lipids-lipoproteins, cholesterol has been the most frequently measured to detect individuals predisposed to CHD. It has been amply documented that hypercholesterolemia is associated with grossly increased risk. The suggestion has been made that risk can be better predicted by measuring levels of serum triglyceride or of lipoproteins. The limited amount of data available do not support this view. Reports from prospective studies indicate that serum cholesterol is at least as good a predictor as any other lipid or lipoprotein measurement, and that none of the others add to the predictive power of cholesterol (7). More research is needed in this area.

At present, particularly for mass screening purposes in communities, serum cholesterol measurement is the procedure of choice, especially since it can be done without fasting, is subject to less laboratory error than the other procedures, and is less expensive. For the physician evaluating an individual patient in the office, it is useful to collect blood after a 15-hour fast (with the last meal low or moderate in fat and free of alcohol), and measure both cholesterol and triglyceride, since-if hyperlipidemia is present-availability of both measurements assists in guiding treatment. In most cases, there is no need for the practitioner routinely to measure serum lipoproteins in addition, whether by ultracentrifuge or electrophoresis. If triglycerides are markedly elevated and cholesterol less so, there is the outside possibility of a rare familial hyperchylomicronemia (either Type I or Type V hyperlipoproteinemia) (8). To rule this out, a satisfactory simple procedure is to inspect the fasting serum 
after it stands overnight in the refrigerator. A cream layer at the top of the scrum indicates abnormal chylomicronemia, provided the patient was really fasting for 15 hours, and his last meal wasn't laden with fat and alcohol. If the infranant is clear, it's a Type I; if cloudy (lipemic) it's a Type V-both rare entities, both treatable by diets low in total fat (less than $10 \%$ of calories from fat) and (especially for Type V) correction of overweight (9).

In the general population, the common phenomenon is "moderate" hypercholesterolemia-either without hypertriglyceridemia, or with hypertriglyceridemia slight moderate or marked but without chylomicronemia. (A reasonable cutting point for fasting serum triglyceride abnormality is 150 $\mathrm{mg} / \mathrm{dl}$ ). Once conditions known to induce hyperlipidemia (e.g., uncontrolled diabetes, hypothyroidism, nephrosis, biliary obstruction, pancreatic disease, alcoholism, myeloma, contraceptive steroids) have been ruled out, it may be concluded that the abnormality is fundamentally diet-induced, i.e., acquired. And it can almost always be alleviated by change in diet habit, i.e., to a calorie-controlled diet low in saturated fat and cholesterol, moderate (not low) in total fat and carbohydrate, moderate (not high) in polyunsaturated fat (7).

To designate the common hyperlipidemia of Americans as acquired is not to say that genetic factors do not operate in them. Since some (a small minority) of Americans maintain very low levels of all serum lipids on usual U. S. diets, there must be an element of host response in all acquired cases of hyperlipidemia, and almost certainly this often reflects inborn (genetic) differences in metabolism. Correspondingly, since almost all persons with familial severe hyperlipidemia respond (at least in part) to diet, their condition is to a degree environmental in origin and related to the usual U. S. diet. Therefore, the distinction between acquired and familial primary hyperlipidemia is relative, not absolute. This conclusion in no way contradicts the fact that among persons with severe hyperlipidemias in particular, disease usually is due predominantly to genetic metabolic abnormalities.

A practical consequence, for both diagnosis and treatment-of this and other risk factors - is the importance of evaluating the entire immediate family. For example, children inherit both their parents' genes and living habits. The latter are amenable to environmental influence. Identification of susceptibility in a parent must, therefore, immediately call attention to his or her children and to parental siblings; the converse is equally true. Hence, prevention, especially early prevention, is an intrinsic part of family medicine. The basic aim is: by changing living habits (the environment) - on a family basis - to control hereditable risk factors and thereby mute or negate genetic predisposition to premature CHD. This is a key aspect of the strategy of focusing on risk factors, to curb the epidemic occurrence of this disease.

Is control of hyperglycemia-along with the major risk factors-an important aspect of this strategy? For years, clinical diabetes has been recognized as a serious risk factor for atherosclerotic disease, although the mechanism is not yet well understood. The association between diabetes and coronary, cerebrovascular, and peripheral vascular disease has been extensively documented in retrospective studies, clinical and pathologic. Other data confirm that persons 
with atherosclerotic disease more often manifest abnormal glucose tolerance than do control subjects $(10,11)$.

Recently population studies have begun to present relevant data in this area. The study of DuPont Company employees, involving an average of more than 73,000 men and women age 25-64, identified 662 persons with diabetes (12). Of these, 418 were normotensive. Their prevalence rate of CHD was $39 \%$ higher than that of randomly selected normotensive nondiabetics - but the difference was not statistically significant. In contrast, the 244 hypertensive diabetics had a CHD prevalence rate nearly double that of hypertensive controls - and the difference was highly significant statistically. The question arises: Is diabetes an independent CHD risk factor? In a more recent report from this study, additional data are presented indicating that clinical diabetes, at least, is an independent risk factor (29).

With respect to asymptomatic hyperglycemia (as distinct from clinical diabetes), the Tecumseh study - based on response at one hour to a $100 \mathrm{~g}$ oral glucose load given in its first round of examinations - reported an association between hyperglycemia and CHD prevalence for both men and women (13). The finding was independent of and additive to the effect of serum cholesterol and blood pressure. Thus far, this is the only set of data indicating that chemical diabetes adds independently to risk. Its suggestive positive findings cannot be regarded as resolving the issue, pending both incidence data and replication in another study. (The problem in interpreting CHD prevalence data is a well-known bugbear of cardiovascular epidemiology.) The Tecumseh study has also reported that its long-term follow-up observations show an association between hyperglycemia and incidence of CHD (14). However, no data are available as yet to clarify whether this relationship is independent of other risk factors. Results from the Framingham study-where the measurement was a casual blood glucose determination, without a glucose load-suggest that hyperglycemia is not an independent risk factor, not for atherosclerotic coronary disease, at least (15).

The only other set of available prospective data is from the study of middleaged men employed by the Peoples Gas Company in Chicago (7). While that investigation was begun in 1958, measurement of glycemia one hour after a 50 $\mathrm{g}$ oral load was done for the first time only in 1965. Several relevant items emerged from the initial set of data: Plasma glucose and blood pressure (both systolic and diastolic) were significantly correlated, and this relationship held up after control for age, relative weight, and thiazide therapy. The higher the level of plasma glucose after load, the higher the blood pressure. Men with frank hyperglycemia (plasma glucose equal to or greater than 205 $\mathrm{mg} / \mathrm{dl}$ - equivalent to whole blood glucose level equal to or greater than 170) had a prevalence rate of hypertension of $32 \%$ compared to $18 \%$ for men with glucose levels less than 205. Hyperglycemic men also exhibited higher rates of hypercholesterolemia and hyperuricemia.

For the period 1965-1970, age-adjusted CHD mortality rate was 42 per 1,000 for the hyperglycemic men, 16 per 1,000 for the normoglycemic men. Most of the excess mortality among hyperglycemic men was of men who 
were also hypertensive. (Hypercholesterolemia and cigarette smoking were also implicated.) Numbers of CHD deaths are small so far in these groups, and further follow-up is needed over the next years to assess the long-term trends. Nevertheless, the data pose important questions: What are the mechanisms-over and above chronic caloric imbalance-of the associations between hyperglycemia and hyperlipidemia, between hyperglycemia and hypertension, between hyperglycemia and hyperuricemia? Is a good deal of the excess atherosclerosis risk of mild maturity-onset nonketotic non-insulindependent diabetics in our population a resultant of the concomitant hypertension and/or hyperlipidemia, and not independently related to the hyperglycemia per se?

The posing of these questions is not an esoteric exercise in epidemiologic dialectics. Very practical issues are involved. Diabetics of this type number about $10,000,000$ in the U.S. A., and their prime problem far-and-away is risk of atherosclerotic "complications." It is now more than ever clear from the results of the University Group Diabetes Program study that treatment of hyperglycemia with drugs-oral or parenteral (insulin)-is ineffective in averting morbidity or mortality from cardiovascular complications in such diabetics (16). Their atherosclerosis is no different in kind-only in degree-from that of nondiabetics. Therefore a total reconsideration and redefinition is needed of control for such diabetics. Even if for practical purposes we assume -or, rather, especially if we assume - that hyperglycemia is an independent and additive CHD risk factor for the diabetic, his risk of premature atherosclerotic disease is also potently related to his blood pressure, serum cholesterol, cigarette smoking - i.e., to the major coronary risk factors. Therefore control cannot remain focused narrowly on blood and urine glucose, but must become comprehensive. It must include effective control of obesity, to correct hyperglycemia and hypertension, as well as attention to fat composition of the diet, to correct hyperlipidemia. And cigarette smoking must also be corrected. Hope for the prevention of atherosclerotic disease in diabetics lies only in such a comprehensive approach to control.

What about hyperuricemia? When present (e.g., serum uric acid levels of $7.0 \mathrm{mg} / \mathrm{dl}$ or greater), it is often associated with other factors such as obesity, hypertension, and hyperglycemia. Evidence is available that hyperuricemia operates as an independent risk factor, but again further clarification is needed.

What then about obesity? For a good many years, it had been taken for granted by most people in medicine that obesity-moderate as well as marked-predisposed to premature CHD. Presumably, obesity acted as an independent risk factor in so doing-although the mechanism was always obscure.

Recently it has become clear that obese persons are more likely to be hypertensive, hyperlipidemic, hyperglycemic, hyperuricemia. Probably these associations reflect cause-and-effect at work, i.e., chronic caloric imbalance somehow contributes to the development of these traits in susceptible people. (How is generally ill-defined.)

But if the obese person is exceptional and is free of these other risk factors, 
is he still at increased risk? The findings to date are equivocal. It appears that in the absence of other risk factors, moderate obesity adds little to risk, but this is still open. Very marked obesity is another matter. In any case, obesity associated with other risk factors is common, and its correction with a diet of optimal fat composition remains an important aspect of controlling the obesity-related risk factors.

\section{HABITUAL DIET HIGH IN CALORIES, SATURATED FATS, CHOLESTEROL}

It is evident-from everything said already - that the habitual diet of Americans may contribute significantly to risk of premature CHD in at least four ways - via hyperlipidemia, hypertension, hyperglycemia and hyperuricemia. This diet-high in saturated fats and cholesterol-encourages the development of hypercholesterolemia in all with any genetic predisposition, i.e., in tens of millions. All too often this diet is also excessive in calories (for level of physical activity), so that obesity develops - and as a frequent consequence, hypertension, hyperlipidemia, hyperglycemia, hyperuricemia. Therefore, this "rich" diet could well be added as a fourth major risk factor to the "big three."

From a practical point of view, therefore, improvement in eating habits is a key part of a rational prophylactic regimen. Obviously, avoiding weight gain - or correcting it - can help significantly to prevent or control hypertension, hyperlipidemia, hyperglycemia and hyperuricemia in an appreciable proportion of the population. And an improved composition of dietary fat intake-as described briefly above, and discussed in detail elsewhere in this Symposium - is the key nutritional step qualitatively in the preventive effort.

\section{SEDENTARY LIFE STYLE (HABITUAL PHYSICAL INACTIVITY, LACK OF EXERCISE, \\ LOW LEVEL OF CARDIOPULMONARY FITNESS)}

Sedentary life style - with resultant poor cardiopulmonary fitness - has also been implicated as a coronary risk factor. While the findings are not as clearcut and consistent as for the major risk factors, they are sufficient to warrant concern in developing preventive programs. (Obviously, physical inactivity is an important contributor to the mass occurrence of obesity in the U. S. A., not only among the middle-aged, but also among young adults, teen agers and children.)

Initial data on physical inactivity as a possible risk factor came from Great Britain: In relatively homogeneous populations of middle-aged men, coronary disease mortality rates over the long term were higher in groups whose work involved relatively little physical activity (bus drivers and telephone operators) than among others more active (bus conductors and postmen) (17); however, there is a suggestion of confounding by preselection of the employee groups, i.e., the bus drivers tended to be more obese than the conductors when they first went to work as young adults (18). On the other hand, a largescale 5-year study of groups of middle-aged European men was nonconfirmatory (4). Conspicuous are the findings on the cohort of Finns, who consumed a 
diet very high in saturated fat and cholesterol, and were generally very active and nonobcsc. The strenuous character of their work apparently gave little or no protection against either hyperlipidemia or CHD, since they were higher on both these parameters than U. S. railroad workers (4).

The most impressive positive data from U. S. studies come from Framingham (19). When five indices of activity and fitness were simultaneously evaluated-a questionnaire-based scale of total daily activity of work and leisure, vital capacity, heart rate, relative weight and hand grip strength-persons assessed as more fit had fewer fatal heart attacks than the less fit. This association was independent of serum cholesterol, blood pressure, and cigarette smoking.

Therefore, light to moderate exercise, of the type enhancing cardiopulmonary fitness (vigorous walking, jogging, bicycling, swimming) may be protective - if approached properly to avoid potential hazards - especially when combined with reasonable alterations in diet and smoking habits.

Tachycardia. For years, analyses by life insurance actuaries have indicated rapid resting heart rate to be one of the "minor impairments" associated with increased risk of cardiovascular and coronary mortality (20). As indicated in the preceding section, the Framingham study has presented data consistent with this conclusion (19). So also has the Western Electric study (6c).

Recently, a detailed analysis of this matter was presented from the Peoples Gas Company study (6l). Resting heart rates of 80 beats/min or greater at initial examination in 1958 were associated with higher 10 -year mortality rates. This relationship of heart rate to risk was apparently independent of-and additive to-the effects of hypercholesterolemia, hypertension, and cigarette smoking.

These data furnish a further rationale for multifactor prevention programs - especially exercise to enhance cardiopulmonary fitness, and cessation of cigarette smoking - since both these measures result in slowing of the heart rate.

Personality-behavior patterns, social incongruities, psychosocial tensions. Psychosocial tensions related to personal life situations and/or those inherent in cultural circumstances have long been suspect as factors related to premature CHD. As was noted in the Report on the Primary Prevention of the Atherosclerotic Diseases of the Inter-Society Commission for Heart Disease Resources, this view has recently received support from several studies (21). For example, data from investigations on "high-drive" personality-behavior patterns and on social incongruities indicate that these may be significant risk factors for premature CHD in our society, independent of and additive to such other traits as "rich" diet, hypertension, hypercholesterolemia, cigarette smoking and lack of exercise $(22,23)$. Similar data have also been obtained indicating a detrimental effect of social and geographical mobility and urbanization (24). These various psychosocial influences may be conditioning and/or aggravating factors in our society; particularly in the presence of other traits enhancing proneness to premature CHD. 
Most of the positive findings indicating associations between various psychosocial factors and premature CHD have been recorded in single investigations and they require confirmation. Obviously this is understandable in view of the complexity of measurement in this area.

Family history of premature atherosclerotic and/or hypertensive disease. Coronary heart disease has long been viewed as related to family history. Evidence exists indicating an increased risk of CHD in close relatives of persons who experience a heart attack early in life, e.g., prior to age $50(25,26)$. There are numerous examples of multiple premature attacks within families. In contrast, there is little evidence for familial aggregation when the disease first occurs late in life. It is likely that much of this predisposition is mediated by familial resemblances in key risk factors, e.g., hypercholesterolemia, hypertension, cigarette smoking (11). Obviously, most of these predisposing influences are under both environmental and genetic control. As already noted, families share not only genes, but also living habits, e.g., "rich" diet, cigarette smoking, sedentary living habit-hence the preventive approach, through early detection and control of risk factors, must be a family affair.

\section{CLINICAL SYMPTOMS AND SIGNS}

Is it reasonable to consider clinical symptoms such as chest discomfort, or signs such as lesser electrocardiographic abnormalities as risk factors? Manifestations of this kind certainly cannot be considered "preclinical" warning signals, because their presence indicates that clinical disease (if only on a suspect level) is already present. Yet persons with seemingly minor manifestations are at increased risk of developing a "major coronary event," as defined previously! In this sense, lesser symptoms and signs - cardiac precursors of major coronary events - can be legitimately considered risk factors $(11,27)$.

Obviously, there is really no sharp line of demarcation between absence and presence of clinically manifest disease. This is well illustrated by stress tests. An abnormal electrocardiographic response to exercise is recorded in a sizeable proportion of middle-aged American men with normal resting ECGs; such men have a several-fold increase in risk of a major coronary event (28). Certainly, therefore, such abnormal responses are properly included in the spectrum of coronary risk factors.

\section{CONCLUSION}

The overwhelming weight of evidence implicating the several risk factors-especially the major risk factors - makes it mandatory for physicians in practice as well as those in public health to incorporate detection in regular examinations of adults, including the asymptomatic. There seems little justification not to do so, especially since early identification of very high risk persons-common in our population-is feasible with use of a few simple, familiar, inexpensive procedures.

In line with what has been learned about the hazards associated with multiple risk factors, the Inter-Society Commission for Heart Disease Resources also urged development and expansion of community programs (using allied 
health personnel as well as physicians) to detect and treat persons of all ages highly susceptible to premature atherosclerotic disease (1). On the basis of recent experience such programs could have a large yield, since fully 20-30\% of middle-aged adults appear to be at very high risk $(1,2)$.

The inference is that with identification of persons at risk by such means appropriate measures can be taken against controllable risk factors. If reversal of atherosclerotic lesions is achievable in primates and other animals by nutritional means (and a similar approach has reportedly caused regression of atherosclerosis in man), surely a prophylactic effort is indicated, with control of the major risk factors its cornerstone. For the great mass of the population who have not yet experienced a clinical coronary event, the bold goal of reversal of lesions need not be set; the more modest ones of stopping or at least markedly slowing atherogenesis will suffice.

As a matter of fact, all the key medical and public health organizations in the United States have agreed for years on the need to find the high risk people (they with their families number in the tens of millions), and bring them under effective long-term care-although precious little has been done so far to implement this accepted policy (1). As far as the U. S. population as a whole is concerned, some argue that in the absence of "final" proof from mass field trials of the relationship of diet to hyperlipidemia and atherosclerotic disease, general nutritional-hygienic recommendations to the public as a preventive measure would lack authenticity. However, is it not wiser (as the InterSociety Commission Report proposed) to use best judgment based on the huge amount of evidence already available, and implement safe measures that can probably stem the epidemic - rather than suspend judgment? Since contemporary living habits render our entire population more or less at risk with respect to this disease, it seems entirely fitting to urge the public at large to modify living habits in the direction of removing or reducing risk of premature coronary disease. Indeed, the Inter-Society Commission emphasized the need and propriety of primary prevention measures for the general public (1). In recommendations aimed at individual physicians, and health professions and policy makers, the Commission called for a three-pronged program: dietary changes to prevent or control hyperlipidemia, obesity, hypertension and diabetes; elimination of cigarette smoking; pharmacologic control of elevated blood pressure.

Emphasis on the high risk individual should not deflect concern from prophylactic measures aimed at protecting the entire population against the large-scale occurrence of premature atherosclerotic disease. Any decision to withhold general recommendations to the public on the ground that "final" proof of their benefit is not yet at hand must also mean continued acceptance of high incidence and mortality rates for coronary heart disease among our young adult and middle-aged populations over the next decades. This toleration of the coronary epidemic is entirely unnecessary and unjustifiable if one accepts, as we believe one must, the scientific evidence as it has been interpreted and applied in this paper. 


\section{ACKNOWLEDGMENTS}

The authors are grateful to the principal investigators of the studies of Albany Civil Servants, Chicago Western Electric Company studies, Framingham, and Minneapolis-St. Paul businessmen for making their data available through the National Cooperative Pooling Project. It is a pleasure to acknowledge the cooperation and aid of our colleagues in this endeavor, Drs. Henry Blackburn, Jøhn M. Chapman, Thomas R. Dawber, Josepher T. Doyle, William B. Kannel, Ancel Keys, Felix J. Moore, Oglesby Paul and Henry L. Taylor.

Appreciation is expressed to Dr. Charles K. Friedberg, Editor-in-Chief, Circulation, for permission to reproduce Figs. 1-4.

\section{REFERENCES}

1. Inter-Society Commission for Heart Disease Resources. Atherosclerosis Study Group and Epidcmiology Study Group. Primary prevention of the atherosclerotic diseases. Circulation 42, A55 (1970).

2. EPSTEIN, F. H. Coronary heart disease epidemiology-current aspects of research, prevention and community programs, in "Trends in Epidemiology, Community Health and Trọpical Medicine” (G. T. Stewart, Ed.). Charles C. Thomas, Springfield, Ill. (in press).

3. TRuett, J., Cornfield, J. AND Kannel, W. A multivariate analysis of the risk of coronary heart disease in Framingham. J. Chron. Dis. 20, 511 (1967).

4. Keys, A. (Ed.) Coronary Heart Disease in Seven Countries. Circulation 41, (Suppl. 1), I-1 (1970).

5. Stamler, J. unpublished data.

6. Data from the Pooling Project, Council on Epidemiology, American Heart Association - a national cooperative project for pooling data from the Albany civil servant, Chicago Peoples Gas Company, Chicago Western Electric Company, Framingham community, Los Angeles civil servant, Minneapolis-St. Paul businessmen, and other prospective epidemiologic studies of adult cardiovascular disease in the United States. The following are representative references on the individual studies and on the results of the Pooling Project presented to date:

6a. DoYle, J. T. Risk factors in coronary heart disease. N. Y. State J. Med. 63, 1317 (1963).

6b. Stamler, J. Cardiovascular diseases in the United States. Amer. J. Cardiol. 10, 319 (1962).

6c. Paul, O., Lepper, M. H., Phelan, W. H., Dupertuis, G. W., MacMillan, A., McKean, H. AND PARK, H. A longitudinal study of coronary heart disease. Circulation 28, 20 (1963).

6d. Dawber, T. W., KanNel, W. B. AND MCNAMARA, P. M. The prediction of coronary heart disease. Trans. Ass. Life Insur. Med. Dir. Amer. 47, 70 (1964).

6e. Chapman, J. M. AND Massey, F. J. The interrelationship of serum cholesterol, hypertension, body weight, and risk of coronary disease. Results of the first ten years follow-up in the Los Angeles Heart Study. J. Chron. Dis. 17, 933 (1964).

6f. Keys, A., Taylor, H. L., Blackburn, H., Brozek, J., Anderson, J. T. and Simonson, E. Coronary heart disease among Minnesota business and professional men followed fifteen years. Circulation 28, 381 (1963).

6g. MOORE, F. E. Some preliminary findings from the Pooling Project of the Council on Epidemiology, American Heart Association. Paper presented at the Conference on Cardiovascular Disease Epidemiology, Council on Epidemiology, American Heart Association, March 3-4, 1969, New Orleans, La.

6h. DoYLe, J. T. AND KINCH, S. H. Coronary heart disease in the United States: Some preliminary findings from the Pooling Project of the Council on Epidemiology of the American Heart Association. Presented at the 42nd Scientific Sessions, American Heart Association, Nov. 14, 1969. 
6i. Epstein, F. H. AND Moore, F. E. Progress Report to the National Heart Institute on the National Cooperative Pooling Project, 1968.

6j. Paul, O. The risks of mild hypertension: A ten year report. Brit. Heart J. 33, (Suppl.), 116 (1971).

6k. DOYLE, J. T. AND KanNEL, W. B. Coronary risk factors: 10 year findings in 7,446 Americans. Pooling Project, Council on Epidemiology, American Heart Association. Paper presented to the VI World Congress of Cardiology, London, England, September, 1970.

61. Berkson, D. M., Stamler, J., Lindberg, H. A., Miller, W. A., Stevens, E. L., Soyugenc, R., Tokich, T. J. AND STAMLER, R. Heart rate: An important risk factor for coronary mortality - ten year experience of the Peoples Gas Co. Epidemiologic Study (1958-68). Jones, R. J., Ed. "Atherosclerosis," Second International Symposium, p. 382, SpringerVerlag, New York, 1970.

7. Stamler, J., Berkson, D. M. AND LindBerG, H. A. Risk factors: Their role in the etiology and pathogenesis of the atherosclerotic diseases. in "Pathogenesis of Atherosclerosis" (R. W. Wissler and J. C. Geer, Eds.), Waverly Press, Baltimore, Maryland (in press).

8. FREDRICKSON, D. S., LEVY, R. I. AND LEES, R. S. Fat transport in lipoproteins - An integrated approach to mechanisms and disorders. N. Eng.J. Med. 276, 34, 94, 148, 215 and 273 (1967).

9. LEES, R. S. AND WILSON, D. E. The treatment of hyperlipidemia. N. Eng. J. Med. 284, 186 (1971).

10. EPSTEIN, F. H. Hyperglycemia: A risk factor in coronary heart disease. Circulation 36, 609 (1967).

11. Epstein, F. H. AND Ostrander, L. D., JR. Detection of individual susceptibility toward coronary disease. Prog. Cardiovasc. Dis. 13, 324 (1971).

12. Pell, S. And D'Alonzo, C. A. Some aspects of hypertension in diabetes mellitus. 202, 10 (1967).

13. Epstein, F. H., Ostrander, L. D., Jr. Johnson, B. C., Payne, M. W., Hayner, N. S., Keller, J. B. AND FranCIS, T., JR. Epidemiological studies of cardiovascular disease in a total community-Tecumseh, Michigan. Ann. Intern. Med. 62, 1170 (1965).

14. Ostrander, L. D., JR. Hyperglycemia and vascular disease in Tecumseh, Michigan. Proceedings of the First International Symposium on Early Diabetes, Marbella, Spain. October 23-26, 1968. Academic Press. Early Diabetes, 1970, p. 365.

15. The Framingham Study. An Epidemiological Investigation of Cardiovascular Disease. (Kannel, W. B. and Gordon, T., editors). Section 27, December 1970. U. S. Government Printing Office, Washington, D. C. 20402.

16. University Group Diabetes Program. The University Group Diabetes Program-A study of the effects of hypoglycemic agents on vascular complications in patients with adult-onset diabetes. Diabetes 19, (Suppl 2) 747 (1970).

17. Morris, J. N., Heady, J. A., Raffle, P. A. B., Roberts, C. G. and Parks, J. W. Coronary heart disease and physical activity of work. Lancet 2, 1053, 1111 (1953).

18. Morris, J. N. Occupation and coronary heart disease. Arch. Intern. Med. 104, 903 (1959).

19. KANNEL, W. B. Habitual level of physical activity and risk of coronary heart disease: The Framingham Study. Can. Med. Ass. J. 96, 811 (1967).

20. Build and Blood Pressure Study, 1959, Vol. I. Society of Actuaries, Chicago, Ill., 1959.

21. JEnkINS, C. D. Psychologic and social precursors of coronary disease. N. Eng. J. Med. 284, 244, 307 (1971).

22. Rosenman, R. H., Friedman, M., Strauss, R., Wurm, M., Jenkins, C. D. and MesSINGER, H. B. Coronary heart disease in the Western Collaborative Group. J.A.M.A., 195, 86, 1966.

23. Shekkelle, R. B., Ostfeld, A. M. And Paul, O. Social status and incidence of coronary heart disease. J. Chron. Dis. 22, 381 (1969).

24. Syme, S. L., Hyman, M. M. And Enterline, P. E. Some social and cultural factors associated with the occurrence of coronary heart disease. J. Chron. Dis. 17, 277 (1964).

25. EPSTFin, F. H. Risk factors in coronary heart disease-Fnvironmental and hereditary influences. Israel J. Med. Sci. 3, 594 (1967). 
26. Stamler, J. "Lectures on Preventive Cardiology." Grune and Stratton, New York, N. Y., 1967.

27. Rose, G. Predicting coronary heart disease from minor symptoms and electrocardiographic findings. Brit. J. Prev. Soc. Med. 25, 94 (1971).

28. DOYLE, J. T. AND KINCH, S. H. The prognosis of an abnormal electrocardiographic stress test. Circulation 41, 545 (1970).

29. Pell, S. AND D’Alonzo, C. A. Factors associated with long-term survival of diabetics. J.A.M.A., 214, 1833, 1970. 